PROCEEDINGS OF THE

AMERICAN MATHEMATICAL SOCIETY

Volume 136, Number 8, August 2008, Pages 2937-2941

S 0002-9939(08)09203-4

Article electronically published on April 7, 2008

\title{
A SHORT PROOF OF GROMOV'S FILLING INEQUALITY
}

\author{
STEFAN WENGER \\ (Communicated by Jon G. Wolfson)
}

\begin{abstract}
We give a very short and rather elementary proof of Gromov's filling volume inequality for $n$-dimensional Lipschitz cycles (with integer and $\mathbb{Z}_{2}$-coefficients) in $L^{\infty}$-spaces. This inequality is used in the proof of Gromov's systolic inequality for closed aspherical Riemannian manifolds and is often regarded as the difficult step therein.
\end{abstract}

\section{INTRODUCTION}

Gromov's well-known systolic inequality in 2 asserts that for any $n$-dimensional closed aspherical Riemannian manifold $\left(M^{n}, g\right)$, the shortest length $\operatorname{sys}_{1}\left(M^{n}, g\right)$ of a non-contractible loop in $M^{n}$ satisfies

$$
\operatorname{sys}_{1}\left(M^{n}, g\right) \leq c_{n} \operatorname{Vol}\left(M^{n}, g\right)^{\frac{1}{n}},
$$

with $c_{n}$ only depending on $n$. The proof of this relies on the following isoperimetric inequality, often regarded as the difficult step in the proof.

Theorem (Gromov [2, 2.3]). Let $X$ be an $L^{\infty}$-space and $n \geq 1$. Then the filling volume of any $n$-dimensional singular Lipschitz cycle $z$ in $X$ with integer or $\mathbb{Z}_{2}$ coefficients satisfies

$$
\operatorname{Fillvol}(z) \leq C_{n} \operatorname{Vol}(z)^{1+\frac{1}{n}}
$$

where $C_{n}$ depends only on $n$.

Here, $X$ is said to be an $L^{\infty}$-space if $X=L^{\infty}(\Omega)$, the space of bounded functions on some set $\Omega$, endowed with the supremum norm. Furthermore, Fillvol $(z)$ is the least volume of an $(n+1)$-dimensional singular Lipschitz chain in $X$ with boundary $z$.

The proof given by Gromov in [2] in fact applies not only to $L^{\infty}$ but to arbitrary Banach spaces and to Riemannian manifolds admitting cone type inequalities and also works for cycles with real coefficients. For the systolic inequality, however, the above theorem is enough. In [4] it was later shown that Gromov's result (for cycles with integer coefficients) extends to the setting of complete metric spaces admitting cone type inequalities. The arguments in 4 rely on the theory of integral currents in metric spaces 1]; these give a generalization of Lipschitz chains (only) with integer coefficients.

Received by the editors March 29, 2007.

2000 Mathematics Subject Classification. Primary 53C23.

Key words and phrases. Systolic inequality, isoperimetric inequality, Lipschitz chains.

(C)2008 American Mathematical Society Reverts to public domain 28 years from publication 
The purpose of this note is to provide a short and rather elementary proof of the above theorem. The main idea stems from [4, but our proof does not use at all the theory of currents. Apart from being less than two pages, it enjoys the following features different from [2]: Firstly, it works also (and without modification) for CAT(0)-spaces, i.e. simply connected geodesic metric spaces of non-positive curvature in the sense of Alexandrov. Secondly, it does not use the Federer-Fleming isoperimetric inequality in $\mathbb{R}^{N}$ and in fact yields a new proof for the isoperimetric inequality in Euclidean space.

\section{Singular Lipschitz CHAins}

Recall from [2] that a singular Lipschitz $n$-chain is a formal finite sum $c=$ $\sum_{i=1}^{k} m_{i} \varphi_{i}$, where the $\varphi_{i}$ are Lipschitz maps $\varphi_{i}: \Delta^{n} \rightarrow X$ and the $m_{i}$ values in $\mathbb{Z}$ or $\mathbb{Z}_{2}$. Here $\Delta^{n} \subset \mathbb{R}^{n+1}$ is the standard $n$-simplex. The boundary $\partial c$ is by definition the singular Lipschitz chain $\partial c=\sum_{i=1}^{k} m_{i} \partial \varphi_{i}$, where $\partial \varphi_{i}$ is the $(n-1)$-chain induced by restricting $\varphi_{i}$ to the faces of $\Delta^{n}$. The volume of $c$ is $\operatorname{Vol}(c):=\sum\left|m_{i}\right| \operatorname{Vol}\left(\varphi_{i}\right)$, where $\operatorname{Vol}\left(\varphi_{i}\right)$ is the "parametrized" volume of $\varphi$. In the setting of Banach spaces there exists more than one suitable definition of volume. Therefore, $\operatorname{Vol}\left(\varphi_{i}\right)$ may be chosen to be the parametrized Hausdorff, the HolmesThompson, or Gromov's mass* volume. Since all these volumes agree up to a factor at most $n^{n / 2}$ the choice of volume is irrelevant for the proof of the isoperimetric inequality. In what follows singular Lipschitz $n$-chains will simply be called $n$ chains.

For the proof of the isoperimetric inequality, the three basic facts listed below will be needed. There exist constants $A_{n} \geq 2^{-1} n^{-n / 2} \omega_{n}$ and $D_{n}, E_{n}$ depending only on $n$ and the chosen volume such that the three properties below hold $\left(\omega_{n}\right.$ denotes the volume of the unit ball in $\left.\mathbb{R}^{n}\right)$ : Let $X$ be a metric space and $z$ an $n$-cycle in $X$.

Coarea formula. Given a Lipschitz function $\varrho: X \rightarrow \mathbb{R}$ with Lipschitz constant 1 , then

$$
\operatorname{Vol}_{n-1}(z \cap\{\varrho=r\}) \leq E_{n} \frac{d}{d r} \operatorname{Vol}_{n}(z \cap\{\varrho \leq r\})
$$

for almost every $r \in \mathbb{R}$. Furthermore, $z \cap\{\varrho=r\}$ is an $(n-1)$-cycle for almost every $r \in \mathbb{R}$ (after a minor "smoothening" of $\{\varrho=r\}$ ).

Cone inequality. If $X$ is a $\operatorname{CAT}(0)$ or a Banach space and $x_{0} \in X$, then the $(n+1)$-chain $c$ in $X$ obtained by joining each point in $z$ by the geodesic line (respectively, straight line if $X$ is a Banach space) with $x_{0}$ satisfies $\partial c=z$ and

$$
\operatorname{Vol}_{n+1}(c) \leq D_{n} R \operatorname{Vol}_{n}(z),
$$

where $R$ is the smallest number such that $z$ is contained in the ball of radius $R$ around $x_{0}$.

Lower density estimate. For almost every $x$ in the support of $z$,

$$
\liminf _{r \rightarrow 0^{+}} \frac{1}{r^{n}} \operatorname{Vol}_{n}(z \cap B(x, r))>A_{n} .
$$

If $X$ is a Banach space and Vol is Gromov's mass* volume, then $D_{n} \leq 1$ and $E_{n}=1$. If $X$ is a CAT(0)-space, then all the definitions of volume agree and we have $D_{n}=\frac{1}{n+1}$ and $E_{n}=1$. 
Gromov's proof [2] uses the coarea formula and the cone inequality, but not the lower density estimate (which is false for chains with real coefficients). Note that (2.3) is clear when $z$ is a piecewise $C^{1}$-cycle. In our arguments this will be enough (by a "smoothening" argument). For Lipschitz cycles the proof is given in [3].

\section{The ShORT PROOF}

Fix a definition of volume, let $n \geq 1$ and let $A_{n}, D_{n}, E_{n}$ be the corresponding values from Section 2, We abbreviate $\mathrm{Vol}=\mathrm{Vol}_{n}$. Let $0<\varepsilon<1$ be given.

Proposition. Let $X$ be a metric space and let $z$ be an $n$-cycle in $X, n \geq 2$. There exist finitely many pairwise disjoint closed balls $B_{i} \subset X, i=1, \ldots, k$, with the following properties:

(1) The volume of $z$ contained in each ball is "not too small":

$$
\operatorname{Vol}\left(z \cap B_{i}\right) \geq 4^{-n} A_{n} \varepsilon \operatorname{diam}\left(B_{i}\right)^{n} .
$$

(2) The restriction $z \cap B_{i}$ is an $n$-chain whose boundary has "small" volume:

$$
\operatorname{Vol}_{n-1}\left(\partial\left(z \cap B_{i}\right)\right) \leq E_{n}\left(A_{n} \varepsilon\right)^{\frac{1}{n}} n \operatorname{Vol}\left(z \cap B_{i}\right)^{\frac{n-1}{n}} .
$$

(3) An essential part of the volume of $z$ is contained in the union of these balls:

$$
\operatorname{Vol}\left(z \cap \bigcup B_{i}\right) \geq \frac{1}{5^{n}} \operatorname{Vol}(z) .
$$

Proof. For $x \in \operatorname{spt} z$ and $r \geq 0$ define $V(x, r):=\operatorname{Vol}(z \cap B(x, r))$ and

$$
r_{0}(x):=\max \left\{r \geq 0: V(x, r) \geq A_{n} \varepsilon r^{n}\right\} .
$$

Note that $0<r_{0}(x)<\infty$ for almost every $x \in \operatorname{spt} z$ by the lower density estimate; moreover,

$$
V\left(x, 5 r_{0}(x)\right)<5^{n} A_{n} \varepsilon r_{0}(x)^{n}=5^{n} V\left(x, r_{0}(x)\right) .
$$

By the Vitali $5 r$-covering lemma there exist finitely many points $x_{1}, \ldots, x_{k} \in \operatorname{spt} z$ such that the balls $B\left(x_{i}, 2 r_{0}\left(x_{i}\right)\right)$ are pairwise disjoint, the balls $B\left(x_{i}, 5 r_{0}\left(x_{i}\right)\right)$ cover spt $z$ and

$$
\operatorname{Vol}\left(z \cap \bigcup B\left(x_{i}, r_{0}\left(x_{i}\right)\right)\right) \geq \frac{1}{5^{n}} \operatorname{Vol}(z)
$$

Fix $i \in\{1, \ldots, k\}$. By the definition of $r_{0}\left(x_{i}\right)$ there exists a non-negligible set of points $r \in\left(r_{0}\left(x_{i}\right), 2 r_{0}\left(x_{i}\right)\right)$ with

$$
\frac{d}{d r} V\left(x_{i}, r\right)<\left(A_{n} \varepsilon\right)^{\frac{1}{n}} n V\left(x_{i}, r\right)^{\frac{n-1}{n}}
$$

therefore, by the coarea inequality,

$$
\begin{aligned}
\operatorname{Vol}_{n-1}\left(\partial\left(z \cap B\left(x_{i}, r\right)\right)\right. & =\operatorname{Vol}_{n-1}\left(z \cap\left\{x: d\left(x, x_{i}\right)=r\right\}\right) \\
& <E_{n}\left(A_{n} \varepsilon\right)^{\frac{1}{n}} n \operatorname{Vol}\left(z \cap B\left(x_{i}, r\right)\right)^{\frac{n-1}{n}} .
\end{aligned}
$$

Choose an $r$ such that the above inequality holds and set $B_{i}:=B\left(x_{i}, r\right)$. The so-defined $B_{i}$ clearly satisfy (1), (2) and (3).

Now let $X$ be a $\operatorname{CAT}(0)$ or an $L^{\infty}$-space. 
Proof of Gromov's theorem. The proof is by induction on $n$ and the case $n=1$ is trivial, since the diameter of a closed curve is bounded by its length and thus the isoperimetric inequality is a direct consequence of the cone inequality. Suppose now that $n \geq 2$ and that the statement of the theorem holds for $(n-1)$-cycles with some constant $C_{n-1} \geq 1$. Set

$$
\varepsilon:=\min \left\{\frac{1}{4^{n-1} C_{n-1}^{n-1} A_{n} E_{n}^{n} n^{n}}, \frac{1}{2}\right\}
$$

let $z$ be an $n$-cycle in $X$ and choose a ball $B$ of finite radius that contains $z$. Let $B_{1}, \ldots, B_{k}$ be balls as in the proposition. By the isoperimetric inequality in dimension $n-1$ we can choose for each $i=1, \ldots, k$ an $n$-chain $c_{i}$ satisfying $\partial c_{i}=\partial\left(z \cap B_{i}\right)$ and

$$
\operatorname{Vol}\left(c_{i}\right) \leq C_{n-1} \operatorname{Vol}_{n-1}\left(\partial\left(z \cap B_{i}\right)\right)^{\frac{n}{n-1}} \leq \frac{1}{4} \operatorname{Vol}\left(z \cap B_{i}\right) .
$$

Here the second inequality follows from (2) and the definition of $\varepsilon$. We may of course assume that $c_{i}$ is contained in $B_{i}$ since otherwise we can project it to $B_{i}$ via a 1Lipschitz projection $P: X \rightarrow B_{i}$ (and this decreases the volume). If $X=L^{\infty}(\Omega)$, then $P(f)(y):=\operatorname{sgn}(f(y)) \min \{|f(y)|, 1\}$. If $X$ is a CAT(0)-space, then $P$ is the orthogonal projection onto $B_{i}$. Set $\hat{z}_{i}:=\left(z \cap B_{i}\right)-c_{i}$ and

$$
z^{\prime}:=z-\sum_{i=1}^{k} \hat{z}_{i}=\left(z \cap\left(\bigcup B_{i}\right)^{c}\right)+\sum_{i=1}^{k} c_{i} .
$$

Observe that these are $n$-cycles and that, by (3.1),

$$
\frac{3}{4} \operatorname{Vol}\left(z \cap B_{i}\right) \leq \operatorname{Vol}\left(\hat{z}_{i}\right) \leq \frac{5}{4} \operatorname{Vol}\left(z \cap B_{i}\right) .
$$

From the proposition and from (3.1), (3.2) we conclude

$$
\operatorname{diam}\left(\hat{z}_{i}\right) \leq \operatorname{diam} B_{i} \leq \frac{4}{\left(A_{n} \varepsilon\right)^{\frac{1}{n}}} \operatorname{Vol}\left(z \cap B_{i}\right)^{\frac{1}{n}} \leq\left(\frac{4^{n+1}}{3 A_{n} \varepsilon}\right)^{\frac{1}{n}} \operatorname{Vol}\left(\hat{z}_{i}\right)^{\frac{1}{n}}
$$

and

$$
\frac{3}{5}\left[\sum_{i=1}^{k} \operatorname{Vol}\left(\hat{z}_{i}\right)\right]+\operatorname{Vol}\left(z^{\prime}\right) \leq \operatorname{Vol}(z)
$$

as well as $\operatorname{Vol}\left(z^{\prime}\right) \leq\left(1-\frac{3}{4} 5^{-n}\right) \operatorname{Vol}(z)$. After further decomposing $z^{\prime}$ as above we may assume that $\operatorname{Vol}\left(z^{\prime}\right)$ is as small as we want. Let $\hat{c}_{i}$ and $c^{\prime}$ be $(n+1)$-chains with boundaries $\hat{z}_{i}$ and $z^{\prime}$, respectively, and which satisfy the cone inequality. Note that we can make $\operatorname{Vol}\left(c^{\prime}\right)$ as small as we wish because $\operatorname{diam} z^{\prime} \leq \operatorname{diam} B<\infty$. The $(n+1)$-chain $c:=\hat{c}_{1}+\cdots+\hat{c}_{k}+c^{\prime}$ has boundary $z$ and satisfies

$$
\operatorname{Vol}(c) \leq \operatorname{Vol}\left(\hat{c}_{1}\right)+\cdots+\operatorname{Vol}\left(\hat{c}_{k}\right)+\operatorname{Vol}\left(c^{\prime}\right) \leq C_{n} \operatorname{Vol}(z)^{1+\frac{1}{n}}
$$

for some $C_{n}$ only depending on $n$. This is a consequence of (3.3), (3.4), and the fact that for $a_{1}, \ldots, a_{k} \geq 0$ and $\alpha \geq 1$,

$$
a_{1}^{\alpha}+\cdots+a_{k}^{\alpha} \leq\left(a_{1}+\cdots+a_{k}\right)^{\alpha} .
$$

This concludes the proof. 
By evaluating the expressions in the proof one sees that the constant $C_{n}$ is bounded above by

$$
C_{n} \leq 27 n D_{n} E_{n} C_{n-1}^{\frac{n-1}{n}} .
$$

Therefore, if Vol is Gromov's mass* volume, the isoperimetric constant is bounded from above by $27^{n} n$ !. Note that this is smaller than the constant that appears in [2.

Clearly, our proof works for all metric spaces with cone inequality for which there exist 1-Lipschitz projections onto closed balls.

\section{ACKNOWLEDGMENTS}

The author thanks Misha Katz for encouraging him to write up this note. The paper was written while the author was visiting the Max Planck Institute of Mathematics in Bonn.

\section{REFERENCES}

[1] L. Ambrosio, B. Kirchheim: Currents in metric spaces, Acta Math. 185 (2000), no. 1, 1-80. MR.1794185 (2001k:49095)

[2] M. Gromov: Filling Riemannian manifolds, J. Differential Geom. 18 (1983), no. 1, 1-147. MR697984 (85h:53029)

[3] B. Kirchheim: Rectifiable metric spaces: local structure and regularity of the Hausdorff measure, Proc. Amer. Math. Soc. 121 (1994), no. 1, 113-123. MR1189747 (94g:28013)

[4] S. Wenger: Isoperimetric inequalities of Euclidean type in metric spaces, Geom. Funct. Anal. 15 (2005), no. 2, 534-554. MR2153909 (2006d:53039)

Courant Institute of Mathematical Sciences, 251 Mercer Street, New York, New YORK 10012

E-mail address: wenger@cims.nyu.edu 